\title{
Protein-loaded chitosan nanoparticles modulate uptake and antigen presentation of hen egg-white lysozyme by murine peritoneal macrophages
}

\section{S. Madrigal-Carballo*, M. Esquivel and \\ M. Sibaja}

Polymers Research Laboratory,

Chemistry School,

National University,

86-3000 Heredia, Costa Rica

E-mail: smadriga@una.ac.cr

E-mail: nesquive@una.ac.cr

E-mail: rsibaja@una.ac.cr

*Corresponding author

\section{J. Vega-Baudrit}

National Nanotechnology Center, CeNAT,

1174-1200 San José, Costa Rica

E-mail: jvegab@cenat.ac.cr

\begin{abstract}
Chitosan binds to negatively charged tripolyphosphate (TPP) by an electrostatic interaction driven by its positively charged amino group. This interaction allows developing stable nanoparticles suitable as a carrier and controlled release system for drugs and vaccines. We study the effect of chitosan nanoparticles $(\mathrm{CNp})$ on the uptake and antigen presentation of the model protein hen-egg white lysozyme (HEL) to peritoneal macrophages isolated from mice. Results showed that after four hours of pre-incubation with a T-cell hybridoma line cocultured with murine peritoneal macrophages, only trace amounts of IL-2 were detected in treatments with HEL alone, whereas cocultures treated with HEL-CNp had already reached maximum IL-2 expression. Confocal microscopy studies showed that $\mathrm{CNp}$ had a higher uptake rate of the fluorescently labelled protein than the protein itself after $30 \mathrm{~min}$ of incubation with the peritoneal macrophages. Our results suggest that $\mathrm{CNp}$ system is a potential candidate for an oral vaccine delivery system.
\end{abstract}

Keywords: chitosan; hen egg-white lysozyme; HEL; immunoadjuvants; nanoparticles; vaccines.

Reference to this paper should be made as follows: Madrigal-Carballo, S., Esquivel, M., Sibaja, M. and Vega-Baudrit, J. (2010) 'Protein-loaded chitosan nanoparticles modulate uptake and antigen presentation of hen egg-white lysozyme by murine peritoneal macrophages', Int. J. Nanoparticles, Vol. 3, No. 2, pp.179-191.

Biographical notes: S. Madrigal-Carballo is a Professor and Vice-Chair in the School of Chemistry at the National University, Heredia, Costa Rica. He received his $\mathrm{PhD}$ in Colloidal and Polymer Physical Chemistry at the University of Valencia, Spain and also completed a Post-Doctoral Fellowship 
at the University of Wisconsin-Madison, USA. Currently, he is working on the development of biomaterials and nanomedicines based on biopolymers isolated from agriculture and fishery waste streams, with special interest in nanoparticles, liposomes and polymer therapeutics.

M. Esquivel is a Professor in the Polymers Research Laboratory at the National University, Heredia, Costa Rica. She received her MSc in Cellulose Chemistry at the University of Guadalajara, Mexico. Currently, she is involved in research on paper aging and biopolymers for pharmaceutical applications.

M. Sibaja is a Professor and Head of the Polymers Research Laboratory at the National University, Heredia, Costa Rica. She received her MSc in Polymer Science at the University of Fukui, Japan. Currently, she is involved in research on biopolymers and biomaterials for agriculture and biomedicine, developing scaffolds for tissue engineering and drug delivery systems based on natural polymers.

J. Vega-Baudrit is a Professor at the National University, Heredia, Costa Rica and also the Head of the National Nanotechnology Center, San José, Costa Rica. His research involves nanotechnology from carbon nanotubes for nanoengineering to nanoparticles for drug delivery, including nanocomposite development for water-based adhesives based on nanosilica, polyurethanes and dextrines.

\section{Introduction}

Novel vaccine adjuvants and particle-based delivery vehicles are being evaluated in a variety of vaccines, including those against diseases such as cancer, malaria, AIDS and hepatitis, among others (Peek et al., 2008; Gupta and Siber, 1995; Mishra et al., 2007; Khatri et al., 2008; Saupe et al., 2006), in which a cellular and/or mucosal immune response is desired.

The development of safe, novel adjuvants is necessary to maximise the efficacy of new and/or available vaccines. According to Gupta and Siber (1995), an 'ideal' adjuvant would elicit a persistent, high quality immune response to an antigen while being non-toxic, biodegradable, non-immunogenic and chemically defined for reproducible manufacture.

Chitosan is an abundant, natural linear polysaccharide derived by the deacetylation of chitin from crustaceans, insects and fungi (Illum, 1998; Singla and Chawla, 2001). Chitosan is non-toxic (LD50 > $16 \mathrm{~g} / \mathrm{kg}$, Arai et al., 1968), biodegradable (Onishi and Machida, 1999), non-immunogenic (Illum, 1998) and can be manufactured reproducibly in accordance with GMP guidelines.

Chitosan's biodegradability, immunological activity and bioadhesion, make it an excellent candidate as a depot/adjuvant for vaccination. Over 20 years ago, chitin derivatives, including chitosan, were found to be potent activators of macrophages and NK cells (Nishimura et al., 1984, 1986). This immunostimulating activity along with the structural similarities between chitin derivatives and glucans, an immunoadjuvant class of natural polysaccharides, led several scientists to study the adjuvant capabilities of chitosan. Seferian and Martinez found that chitosan particles, formulated in an emulsion with antigen, squalene and Pluronic ${ }^{\circledR}$ L121, gave a prolonged, high antigen-specific 
antibody titre and sensitised animals for antigen-specific DTH responses following an IP injection. Chitosan particles alone offered no enhancement of an adaptive immune response (Seferian and Martinez, 2001). However, because of its mucoadhesive properties, chitosan has also been explored as an adjuvant for mucosal and subcutaneous vaccination (Zaharoff et al., 2007). Intranasal administrations of chitosan solutions have enhanced adaptive immune responses to several antigens (Illum et al., 2001; Van der Lubben et al., 2001). The mechanisms of vaccine enhancement by chitosan are believed to be due to both retention of vaccine in the nasal passages via mucoadhesion and opening of endothelial cell junctions for paracellular transport of vaccine (Van der Lubben et al., 2001). Recent clinical studies have confirmed that chitosan is a promising adjuvant platform for intranasal vaccination (Read et al., 2005; Mills et al., 2003; McNeela et al., 2004).

Due to the high protein binding properties of some types of chitosan microparticles, they are also potential candidates for oral delivery of antigens (Zaharoff et al., 2007). Mild preparation can protect the proteins when they are incorporated during preparation of the microparticles (Calvo et al., 1997; Berthold et al., 1996). In order to circumvent protein denaturation conditions, chitosan microparticles can be loaded passively (Jameela et al., 1998).

Macrophages are effector cells of innate immunity and link innate immune responses to acquired immunity through antigen presentation to memory lymphocytes. We tested the effects of protein-loaded $\mathrm{CNp}$ on macrophage antigen presentation of hen egg-white lysozyme (HEL) by mouse peritoneal macrophages to a T-cell hybridoma line that responds to the HEL antigen. The method measures production of interlukin-2 (IL-2) by a T-cell hybridoma line cocultured with murine peritoneal macrophages in the presence of HEL. We also study the effect of $\mathrm{CNp}$ on macrophage uptake through microscopy of the fluorescently labelled protein by direct fluorescence measurement (Jones et al., 1997).

\section{Experimental section}

\subsection{Materials and reagents}

All reagents were analytical grade or better. Chitosan from shrimp shells (degree of deacetylation $95 \%$, calculated by ${ }^{1} \mathrm{HRMN}$; Mw $185 \mathrm{kDa}$ calculated by specific viscosimetry) was provided by the Polymers Research Laboratory (POLIUNA), National University, Costa Rica. Hen egg-white lysozyme (HEL, 95\% protein, Mw $14.7 \mathrm{kDa}$ ) and sodium triphosphate pentabasic (TPP, practical grade, 90\%-95\%) were obtained from Sigma-Aldrich (St. Louis, MO, USA) and used without further purification.

\subsection{Preparation of chitosan nanoparticles ( $\mathrm{CNp})$}

$\mathrm{CNp}$ were prepared based on the ionotropic gelation of chitosan with tripolyphosphate (TPP) anions. Briefly, chitosan was dissolved in acetic acid $1.00 \% \mathrm{v} / \mathrm{v}$ to obtain concentrations ranging $0.05 \%-0.50 \% \mathrm{w} / \mathrm{v}$. TPP was dissolved in water to a concentration of $1.00 \mathrm{mg} / \mathrm{mL}$. Under magnetic stirring at room temperature, $2 \mathrm{ml}$ of TPP solution were added dropwise to $5 \mathrm{ml}$ of chitosan solution. The mixture was stirred for 60 min followed by sonication. The resulting suspension was subsequently centrifuged at $12,000 \times \mathrm{g}$ for 15 min. The precipitate was suspended in water, centrifuged again and then freeze-dried. 
Then the freeze-dried $\mathrm{CNp}$ were resuspended in deionised water for further characterisation and cell culture experiments.

\subsection{Apparent hydrodynamic diameter and $\zeta$-potential}

Apparent hydrodynamic diameter determinations and electrophoretic mobility measurements were carried out with a Zetasizer 2000 (Malvern Instruments, London, UK) and also with a Z-Meter System 3.0 (Z-Meter Inc., Santa Clara, CA, USA) equipped with microscope DR model (Carl Zeiss Microimaging Inc., Thornwood, NY, USA) and an electrophoresis cell GT2 type (Z-Meter Inc., Santa Clara, CA, USA). For apparent hydrodynamic diameter and electrophoretic mobility measurements, the samples were obtained as stated above and afterwards diluted to 1:10 with deionised water. Five samples were prepared for each $\mathrm{CNp}$ formulation. For illustrative purposes, approximate $\zeta$-potential values were calculated starting from the Smoluchowski's equation, and using the following values: $\varepsilon_{o}=8.9 \times 10-12 \mathrm{Fm}^{-1}$ and $\varepsilon_{r}=79$.

\subsection{Surface morphology analysis}

$\mathrm{CNp}$ were visualised by atomic force microscopy (AFM, MFP-3D-SA, Asylum Research, Santa Barbara, CA, USA) in the contact mode. Samples dissolved in water were placed onto freshly cleaved mica and dried in air at room temperature. AFM image was performed with a spring contact of $\mathrm{k}=0.03 \mathrm{~N} / \mathrm{m}$ using a nanoprobe cantilever made of silicon nitride $\left(\mathrm{Si}_{3} \mathrm{~N}_{4}\right)$.

\subsection{HEL loading and release}

HEL was chosen as antigenic model protein. HEL loading of CNp was performed by incubating $\mathrm{CNp}$ (chitosan $0.10 \% \mathrm{w} / \mathrm{v}$ ) and HEL $0.5 \%-2.0 \% \mathrm{w} / \mathrm{v}$ in phosphate buffered saline (PBS; $\mathrm{pH} 7.3$ ) under shaking at $25^{\circ} \mathrm{C}$. After incubation for $180 \mathrm{~min}$, the suspension was centrifuged $(1,400 \mathrm{rpm}$ for $30 \mathrm{~min})$ to remove the unloaded HEL.

The loading degree was determined by quantifying the non-bound HEL in the supernatant with the Lowry protein assay method (Lowry et al., 1951). Both loading capacity (LC) and encapsulation efficacy (EE) were calculated as follows:

$$
\begin{aligned}
& \mathrm{LC}=\left(\mathrm{W} / \mathrm{W}_{\mathrm{n}}\right) \times 100 \\
& \mathrm{EE}=\left(\mathrm{W} / \mathrm{W}_{\mathrm{o}}\right) \times 100
\end{aligned}
$$

where $\mathrm{W}$ is the weight of loaded HEL, $\mathrm{W}_{\mathrm{o}}$ is the total HEL weight and $\mathrm{W}_{\mathrm{n}}$ is the weight of HEL-loaded CNp.

HEL release from CNp was determined in PBS ( $\mathrm{pH} 7.3$ ). To load the systems, $5.0 \mathrm{ml}$ of $\mathrm{CNp} 10 \% \mathrm{v} / \mathrm{v}$ containing $0.5 \%(\mathrm{w} / \mathrm{v}) \mathrm{HEL}$ was incubated for $3 \mathrm{~h}$. After centrifuging $(1400 \mathrm{rpm}$ for $30 \mathrm{~min}$ ) the loaded $\mathrm{CNp}$ were resuspended in $\mathrm{PBS}(\mathrm{pH} 7.3)$ to make a $1.0 \% \mathrm{w} / \mathrm{v}$ suspension. Samples were incubated at $37^{\circ} \mathrm{C}$ under mild shaking. After 15, 30, $45,60,90,120,180$ and $240 \mathrm{~min}$, the tubes were given a spin-off and samples of $500 \mu \mathrm{L}$ of the supernatant were taken and replaced by $500 \mu \mathrm{L}$ of PBS (pH 7.3). The non-bound HEL in PBS was determined with the Lowry et al. (1951) protein assay. 


\subsection{Mouse peritoneal macrophages and T-cell hybridoma cell culture}

In this procedure, six to eight week B10.Br, I-Ak haplotype female mice (cat. no. 000465 , Jackson Laboratories, Bar Harbor, ME, USA) were fed standard chow and housed in the Animal Sciences vivarium. To harvest peritoneal exudative cells, mice were injected with $1.0 \mathrm{ml} \mathrm{3 \%}$ thioglycollate (TG, Sigma B2551) by IP injection. On the fifth day, mice were euthanised with $\mathrm{CO}_{2}$ and sprayed with $70 \%$ ethanol. A small cut was made in the skin covering the peritoneal cavity and the skin was pealed back without tearing the peritoneal membrane. An aliquot of $3.0 \mathrm{ml}$ of phosphate buffered saline (PBS) with heparin $(10 \mathrm{U} / \mathrm{ml})$ was injected $(25 \mathrm{G}$ needle) into the lower right quadrant of the peritoneum. The mouse was shaken gently for 1 minute. A $3.0 \mathrm{ml}$ syringe with 19 gauge needle was used to remove the PBS and cells by inserting the needle into an area that was away from the intestines and slowly aspirating the cells. The exudate was placed in a sterile centrifuge tube on ice and then centrifuged at $800 \times \mathrm{g}$ for five minutes. Cells were washed with $\mathrm{PBS} /$ heparin. If there was red blood cells on the pellet, the blood cells were lysed $(0.17 \mathrm{M}$ TRIS buffer, $\mathrm{pH} 7.4 / 0.16 \mathrm{M} \mathrm{NH}_{4} \mathrm{Cl}$ ), centrifuged and rinsed with PBS. The cells were resuspended in media and plated in 24 well cell culture plates at a concentration of 2 to 5 million cells/well. After two to four hours the monocyte/macrophages were attached and washed with Dubellco's modified essential media (DMEM, no additives) until cells other than macrophages were removed. Supplemented media (DMEM, 10\% fetal bovine serum, $2 \mathrm{mM}$ L-alanyl-L-glutamine, and $100 \mathrm{U} / \mathrm{ml} \mathrm{media} \mathrm{penicillin/100} \mathrm{U/ml} \mathrm{media}$ streptomycin) was added and the macrophages were incubated overnight at $37^{\circ} \mathrm{C}$ and $5 \%$ $\mathrm{CO}_{2}$. The 3A9 T-cell hybridomas were a gift from Prof. Jess D. Reed (University of Wisconsin-Madison, USA) and were maintained in DMEM supplemented with $10 \%$ FBS, 50 ug gentamycin/mL media, 2 mM L-alanyl-L-glutamine, $10 \mathrm{mM}$ HEPES buffer, 55 uM 2-mercaptoethanol, and $1 \times$ non-essential amino acids. For experiments, media was removed from the macrophages and rinsed once with PBS. Media for the macrophages supplemented with HEL-loaded $\mathrm{CNp}(0.0 \%-1.0 \%)$ was added and incubated for $0.25-4$ hours. The media was removed and cells washed once with PBS. The T-cell hybridomas (concentration equals $2 \times$ the number of macrophages) in 3A9 media (without phenol red) were added to the macrophages and incubated ( 24 hours). Media was removed, placed in microfuge tubes, centrifuged for three to four minutes at 10,000 rpm and IL-2 was measured by ELISA kit (cat. no. 555148, B D Biosciences, San Diego, CA, USA). Data was expressed as pg IL-2/ml media. Macrophage cell viability was assessed with a cell counting kit (kit-8, CCK-8, CK04-11, Dojindo Molecular Technologies, Gaithersburg, MD, USA). Viability was based on the reduction of a tetrazolium salt by NADH/NADPH. Upon removal of the 3A9 cells, the macrophages were rinsed once with PBS and $0.4 \mathrm{ml}$ media $(25 \mathrm{uL} \mathrm{CCK}-8 / \mathrm{mL})$ were added and incubated $0.5-2$ hours. The media was removed to a 96 well plate and read at $450 \mathrm{~nm}$ on a plate reader. Data was expressed as absorbance $/ \mathrm{ml}$ media.

\subsection{Microscopy of macrophage endocytosis of antigen-loaded CCL}

Mouse peritoneal macrophages were culture in $35 \mathrm{~mm}$ glass bottom culture plates (P35G-1.0-14-C, MatTek Corp., Ashland, MA 01721) and treated with the HEL-loaded $\mathrm{CNp}$. Endocytosis and subsequent proteolysis of the nanoparticulate system in the endosomes was studied by fluorescent microscopy of HEL labelled by a quenched 
BODIPY dye conjugate (A-20181, Molecular Probes, Eugene, OR, USA). Protein was labelled according to kit instructions and subsequently loaded into the $\mathrm{CNp}$ according to the methodology described above. Macrophages were incubated with the labelled HEL and the HEL-loaded $\mathrm{CNp}$ and $\mathrm{CTNp}$ for $0.25-8$ hours and imaged with a Zeiss fluorescent microscope (Carl Zeiss Microimaging, Thornwood, NY 10594, with $450-490 \mathrm{~nm}$ excitation and 510-565 nm emission filters). Images were captured at a rate of one to 30 minute intervals. The microscope was fitted with a chamber to maintain the cells at $37^{\circ} \mathrm{C}$ and $5 \% \mathrm{CO}_{2}$.

\section{Results and discussion}

\subsection{Characterisation of chitosan (CNp) and protein-loaded chitosan (HEL-CNp) nanopartcles}

Particle apparent hydrodynamic diameter is one of the most significant factors associated with mucosal and epithelial tissue uptake of nanoparticles and in the intracellular trafficking of the particles. Smaller size nanoparticles $(-100 \mathrm{~nm})$ demonstrated more than three-fold greater arterial uptake compared to larger nanoparticles, as the smaller nanoparticles were able to penetrate throughout the sub-mucosal layers while the larger size micron-particles were predominantly localised in the epithelial lining (Gan et al., 2005). The apparent hydrodynamic diameter and $\zeta$-potential variations among the $\mathrm{CNp}$, formulated at different chitosan concentrations and loaded with different antigen concentrations are shown in Table 1.

Table 1 Variation on apparent hydrodynamic diameter (AHD) and $\zeta$-potential for different chitosan $(\mathrm{CNp})$ and HEL loaded (HEL-CNp) nanoparticle formulations

\begin{tabular}{lcc}
\hline Sample ID & AHD $(\mathrm{nm})$ & $\zeta$-Potential $(\mathrm{mV})$ \\
\hline CNp 0.05\% & $130 \pm 25$ & $45.3 \pm 2.9$ \\
CNp 0.10\% & $149 \pm 11$ & $42.9 \pm 1.7$ \\
CNp 0.25\% & $426 \pm 58$ & $30.1 \pm 2.3$ \\
CNp 0.50\% & $1085 \pm 83$ & $25.2 \pm 1.6$ \\
HEL-CNp 0.5\% & $295 \pm 24$ & $40.4 \pm 1.3$ \\
HEL-CNp 1.0\% & $301 \pm 19$ & $38.9 \pm 2.0$ \\
HEL-CNp 2.0\% & $304 \pm 32$ & $37.7 \pm 1.1$ \\
\hline
\end{tabular}

Note: Results are shown as [mean $\pm \mathrm{SD}, \mathrm{n}=5]$.

Results showed an increase in apparent hydrodynamic diameter as a function of the increase in the biopolymer concentration, with a high increase of about $50 \%$ fold in apparent hydrodynamic diameter between the 0.10 and the $0.25 \% \mathrm{w} / \mathrm{v}$ chitosan solutions. The mean apparent hydrodynamic diameter of the $\mathrm{CNp}$ formulated with chitosan $0.5 \%$ $\mathrm{w} / \mathrm{v}$ was around ten times bigger than those formulated with chitosan solutions below $0.10 \% \mathrm{w} / \mathrm{v}$.

The $\zeta$-potential of the $\mathrm{CNp}$ was positive and seems to be proportionally inversed to the concentration of biopolymer. A $\zeta$-potential of $\pm 30 \mathrm{mV}$ is required as a minimum for a 
physical stable nanosuspension solely stabilised by electrostatic repulsion (Muller et al., 2001). The $\zeta$-potential of the $\mathrm{CNp}$ showed values ranging 45 to $25 \mathrm{mV}$, the $\mathrm{CNp}$ formulated with chitosan concentrations higher than $0.10 \% \mathrm{w} / \mathrm{v}$ reached $\zeta$-potential values below the limit for electrostatic stability.

It is well-known that lysozyme is a natural enzyme existing in the human body which can catalyse the hydrolysis of chitinous materials (Ravi Kumar et al., 2004); but also previous reports have shown that degradation rate, due to lysozyme digestion, proved to be affected markedly by the extent of deacetylation degree of chitosan and showed a maximum at 50\% deacetylation (Kurita et al., 2000; Deng et al., 2006); the highly deacetylated samples will exhibit low or no susceptibility to lysozyme degradation, attributable to the decrease in the $\mathrm{N}$-acetylglucosamine sequences crucial as a substrate to be recognised by lysozyme (Varum et al., 1996).

Loading of HEL by the incubation method significantly increased apparent hydrodynamic diameter in comparison to $\mathrm{CNp}$ without protein. The $\zeta$-potential of the HEL-loaded CNp can greatly influence their stability in suspension through electrostatic repulsion between the particles. The effect of HEL loading only slightly decreased the $\zeta$ potential of HEL-CNp and they still show values above the limit for electrostatic stability.

The surface morphology of the CNp observed by atomic force microscopy is shown in Figure 1. The CNp were spherical in shape with a relatively homogeneous apparent hydrodynamic diameter distribution.

Figure 1 Atomic force micrographs 2D (up) and 3D (down) for (A) unloaded chitosan nanoparticles $(\mathrm{CNp})$ formulated with chitosan $0.10 \% \mathrm{w} / \mathrm{v}$ and $(\mathrm{B}) \mathrm{CNp}$ loaded with hen egg-white lysozyme (HEL) $1.0 \% \mathrm{w} / \mathrm{v}$
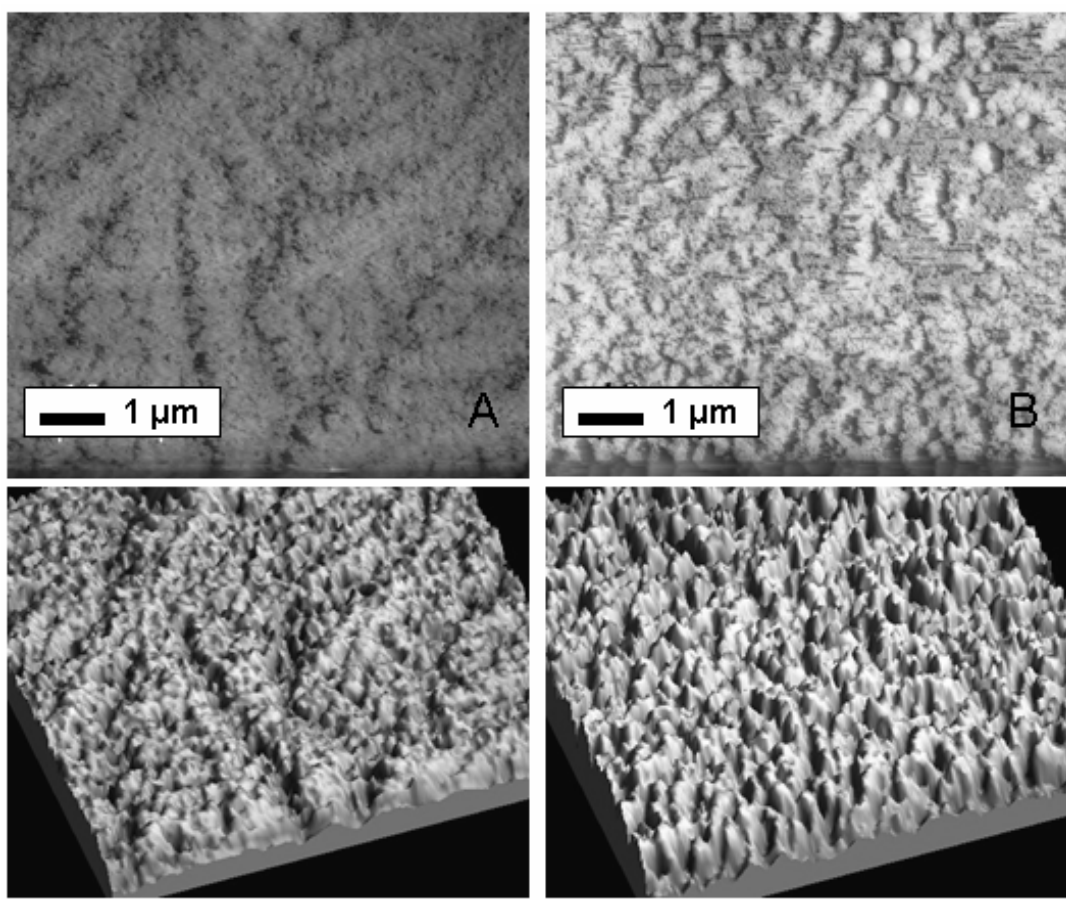


\subsection{Protein loading and release}

The $\mathrm{CNp}$ were loaded with different amounts of HEL by incubation of a $\mathrm{CNp}$ (chitosan $0.10 \% \mathrm{w} / \mathrm{v})$ suspension with $0.5-2.5 \%(\mathrm{w} / \mathrm{v})$ HEL. Both loading capacities (LC) and encapsulation efficacies (EE) were determined as shown in Table 2.

Table 2 Protein loading of chitosan nanoparticles at different concentrations of total HEL (hen egg-white lysozyme)

\begin{tabular}{lcc}
\hline HEL conc. $(\%$ w/v) & Loading capacity $(\%)$ & Encapsulation efficacy $(\%)$ \\
\hline 0.50 & $61.2 \pm 2.3$ & $85.4 \pm 3.6$ \\
1.00 & $58.7 \pm 1.8$ & $76.6 \pm 2.7$ \\
1.50 & $60.3 \pm 2.1$ & $50.8 \pm 1.4$ \\
2.00 & $56.2 \pm 3.5$ & $37.6 \pm 3.1$ \\
\hline
\end{tabular}

Note: Data are expressed as [mean $\pm \mathrm{SD}, \mathrm{n}=5]$.

Results suggest that the LC is not substantially influenced by the amount of HEL available in the loading solution. Thus, the more HEL is offered to the $\mathrm{CNp}$, the more HEL remains unbound during the loading process. Therefore, HEL $1.0 \% \mathrm{w} / \mathrm{v}$ in the loading solution was selected as the optimal concentration. When $\mathrm{CNp}(0.10 \% \mathrm{w} / \mathrm{v})$ were incubated with $1.0 \%$ of HEL, a loading percentage of $(58.7 \pm 1.8) \%(n=5)$ for independently made batches was obtained. Under these conditions, a very high EE of $(76.6 \pm 2.7) \%$ was obtained, indicating that only a small amount of HEL was lost during the loading process.

Figure 2 Protein release profile from HEL loaded-chitosan nanoparticles (HEL 1.0\% w/v, chitosan $0.10 \% \mathrm{w} / \mathrm{v}$ )

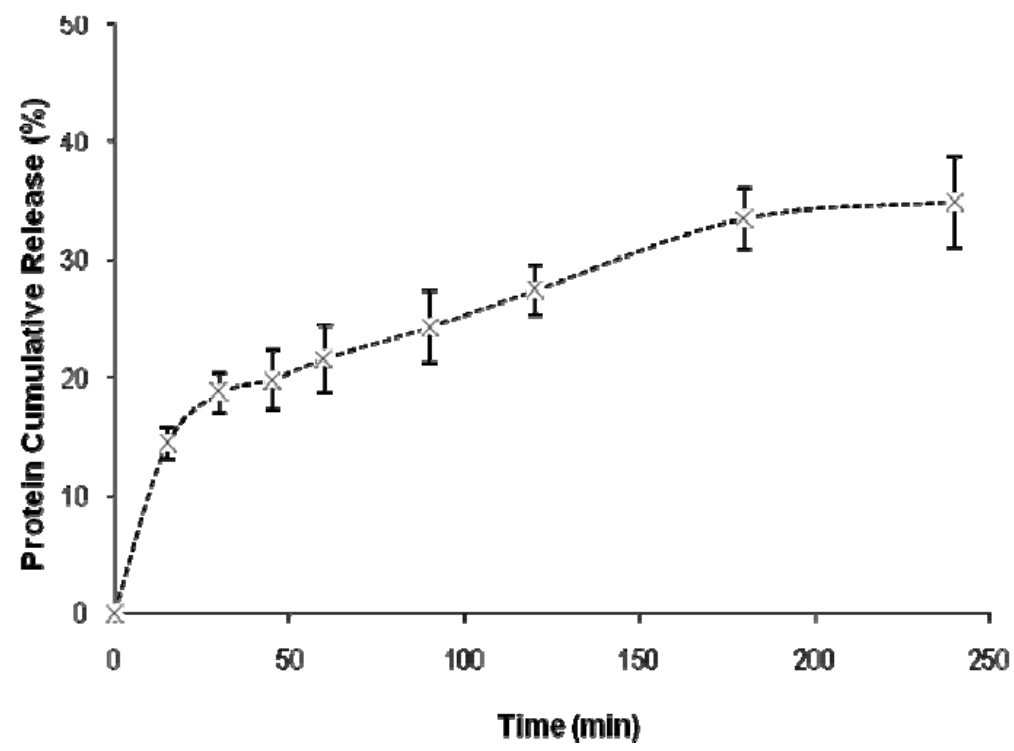

Note: Release medium PBS (pH 7.3), $\mathrm{T}=37^{\circ} \mathrm{C}$. Data are expressed as [mean $\pm \mathrm{SD}$, $\mathrm{n}=3]$. 
Figure 3 Effects of protein-loaded CNp on interlukin-2 (IL-2) expression in 3A9 T-cell hybridomas cocultured with mouse peritoneal macrophages (a) media containing the HEL and HEL-CNp was removed and the T-cell hybridoma culture was added to the macrophages for 24 hours (b) effect of protein concentration for HEL and HEL-CNp on cell viability of mouse peritoneal macrophages

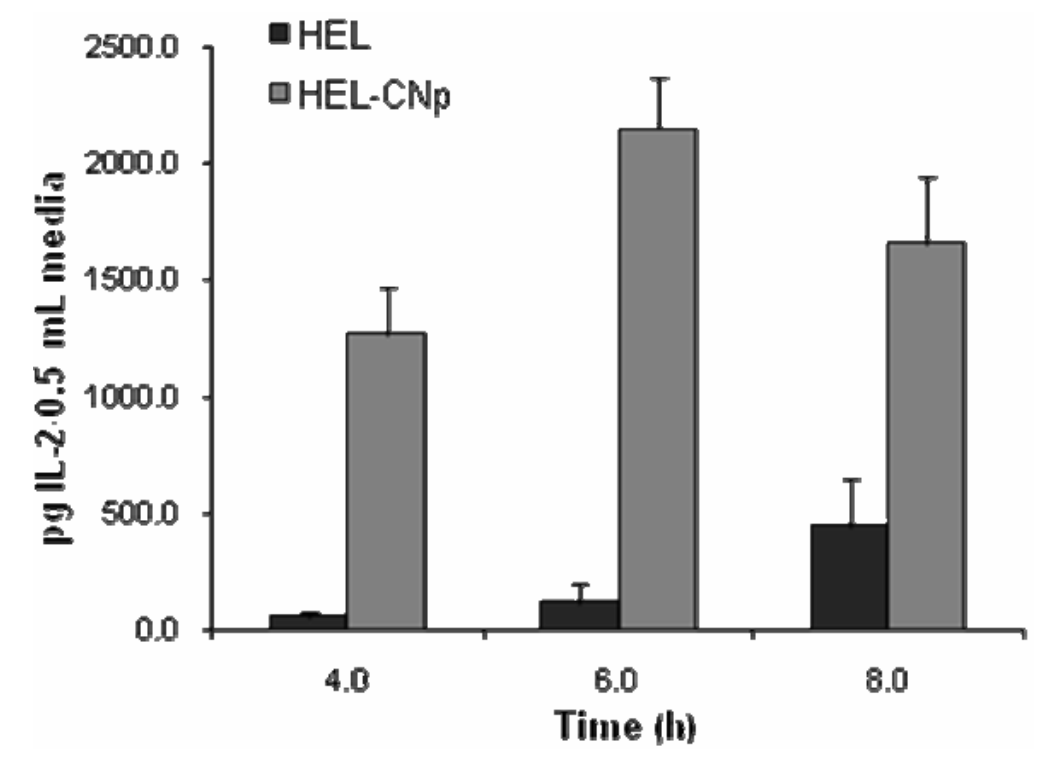

(a)

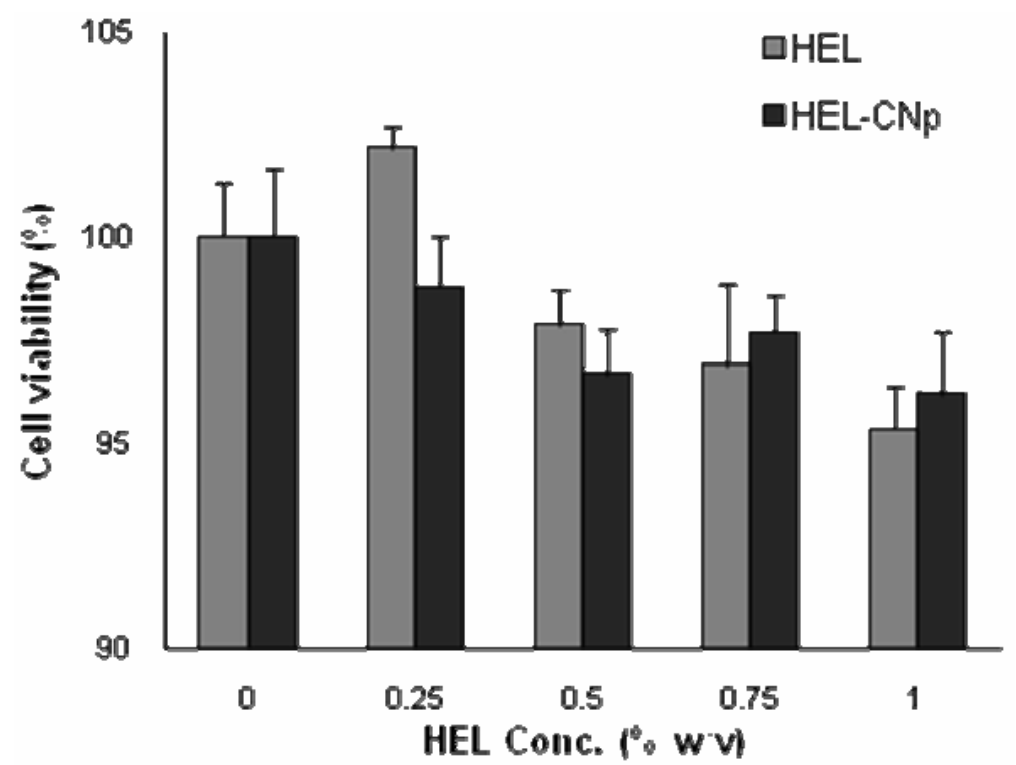

(b)

Note: HEL alone or HEL-loaded CNp were added to the macrophage culture for four, six, and eight hours prior to addition of the T-cell hybridoma. 
Protein release studies in Figure 2 showed that HEL total release at isothermal conditions, decreased significantly when the protein is delivered via $\mathrm{CNp}$. Addition of TPP may provided a greater crosslink density within the biopolymer, causing increased chain packing and rigidity, as well as increased inter-chain bonding, thereby reducing HEL release from the nanoparticle system.

\subsection{Macrophage uptake and antigen presentation}

Figure 3(a) shows the effect of pre-incubation (four, six, and eight hours) of HEL and HEL-loaded $\mathrm{CNp}$ with the macrophages prior to addition of T-cell hybridoma. Media containing nanoparticles was removed prior to addition of the T-cell hybridoma. After four hours of pre-incubation, only trace amounts of IL-2 were detected in the cocultures treated with HEL alone, whereas cocultures treated with HEL-CNp had already reached maximum IL-2 expression. In the absence of $\mathrm{CNp}$, IL-2 expression increased with increasing time of incubation. CNp may increase the uptake of HEL and subsequent expression of IL-2 by the T-cell hybridomas. Alternatively, CNp may decrease rate of HEL proteolysis in the macrophage and allowed more antigenic peptide to be presented to the T-cell hybridoma. Cell culture analysis carried on the peritoneal macrophages after treatment with different concentrations of HEL free and loaded into $\mathrm{CNp}$ showed no significant effects of the nanoparticle system on cell viability [Figure 3(b)].

Figure 4 Hen egg white lysozyme (HEL) was labelled with fluorescent and mouse peritoneal macrophage uptake of HEL alone (A) and HEL-loaded CNp (B) was determined at 30 minutes after adding the antigenic protein $(20 \times)$
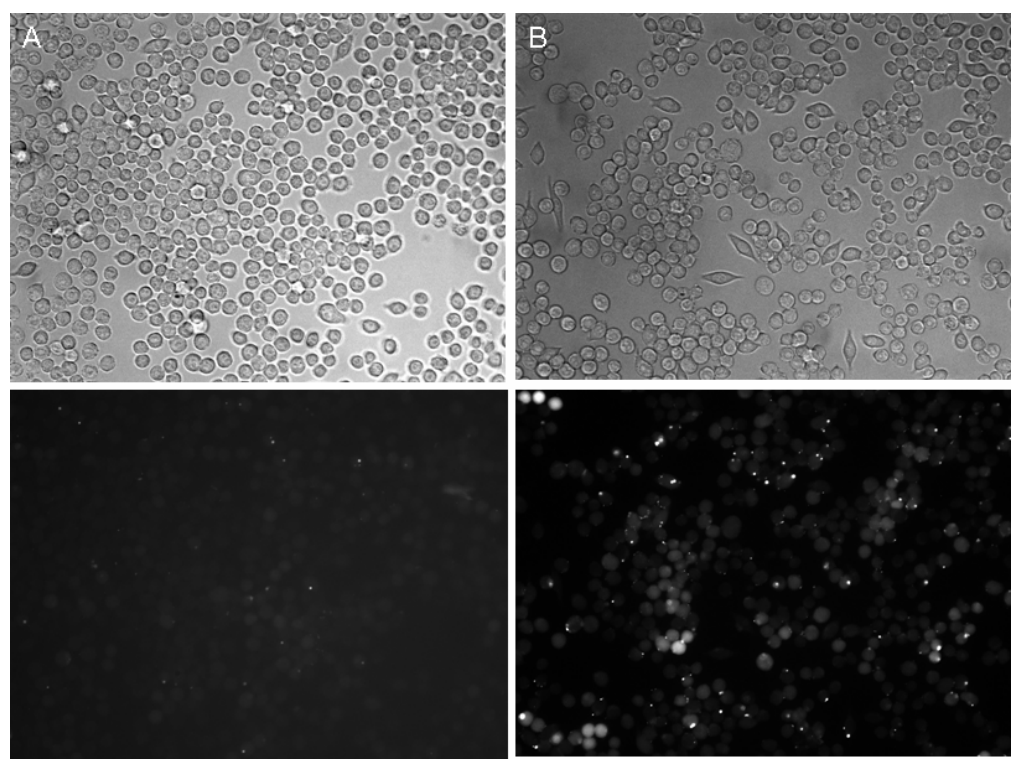

Notes: Phase images on top show the presence of macrophages. Fluorescent images on the bottom show that there is an increased uptake of the fluorescently labelled HEL after $30 \mathrm{~min}$, when protein was delivered by $\mathrm{CNp}$ 
Fluorescently labelled HEL, unloaded and loaded into CNp, was incubated with murine peritoneal macrophages and uptake of the samples was followed by fluorescent microscopy. After 30 minutes, macrophages treated with HEL-CNp clearly contained more fluorescent endosomes than the macrophages treated with HEL alone (Figure 4). Thus, these in vitro studies showed that the delivery of HEL into CNp can increase Peyer's patches macrophage uptake and also modulate antigen presentation.

\section{Conclusions}

Our results indicate that protein-loaded chitosan nanoparticles (HEL-CNp) modulate uptake and antigen processing of the Peyer's patches macrophage cells. This nanostructured system showed high LC and loading efficacy for the model antigenic protein HEL. About $70 \%$ of the HEL remained in the $\mathrm{CNp}$ after release studies for $4 \mathrm{~h}$ in PBS, becoming a suitable system for transportation of the model antigen into the Peyer's patches.

Since uptake by macrophage cells is the first step in oral vaccination, our results suggest that these $\mathrm{CNp}$ are promising as an efficient oral nanovaccine delivery system. Nevertheless, since real vaccines in general provoke stronger immune responses than model antigens like HEL, the CNp system will be further investigated after loading with vaccines against different pathologies.

\section{Acknowledgements}

The authors are deeply thankful with Prof. Jess D. Reed from the University of Wisconsin-Madison for the supply of the 3A9 T-cell hybridomas, as well as Costa Rica's Consejo Nacional de Rectores (CONARE) and Consejo Nacional de Investigaciones Cientificas y Tecnologicas (CONICIT) for the funding of this research.

\section{References}

Arai, K., Kinumaki, T. and Fujita, T. (1968) Bulletin of Tokai Regional Fisheries Research Laboratory, Vol. 43, p.89.

Berthold, A., Cremer, K. and Kreuter, J. (1996) 'Preparation and characterization of chitosan microspheres as drug carrier for prednisolone sodium phosphate as model for anti-inflammatory drugs', Journal of Controlled Release, Vol. 39, pp.17-25.

Calvo, P., Remunan-Lopez, C., Vila-Jato, J.L. and Alonso, M.J. (1997) 'Novel hydrophilic chitosan-polyethylene oxide nanoparticles as protein carriers', Journal of Applied Polymer Science, Vol. 16, pp.125-132.

Deng, Q.Y., Zhou, C.R. and Luo, B.H. (2006) 'Preparation and characterization of chitosan nanoparticles containing lysozyme', Pharmaceutical Biology, Vol. 44, pp.336-342.

Gan, Q., Wang, T., Cochrane, C. and McCarron, P. (2005) 'Modulation of surface charge, particle size and morphological properties of chitosan-TPP nanoparticles intended for gene delivery', Colloids and Surfaces B: Biointerfaces, Vol. 44, pp.65-73.

Gupta, R.K. and Siber, S.R. (1995) 'Adjuvants for human vaccines-current status, problems and future prospects', Vaccine, Vol. 13, pp.1263-1276. 
Illum, L. (1998) 'Chitosan and its use as a pharmaceutical excipient', Pharmaceutical Research, Vol. 15, pp.1326-1331.

Illum, L., Jabbal-Gill, I., Hinchcliffe, M., Fisher, A.N. and Davis, S.S. (2001) 'Chitosan as novel nasal delivery system for vaccines', Advanced Drug Delivery Reviews, Vol. 51, pp.81-96.

Jameela, S.R., Kumary, T.V., Lal, A.V. and Jayakrishnan, A. (1998) 'Progesterone-loaded chitosan microspheres: a long acting biodegradable controlled delivery system', Journal of Controlled Release, Vol. 52, pp.17-24.

Jones, L.J., Upson, R.H., Haugland, R.P., Panchuk-Voloshina, N., Zhou, M. and Haugland, R.P. (1997) 'Quenched BODIPY dye-labeled casein substrates for the assay of protease activity by direct fluorescence measurement', Analytical Biochemistry, Vol. 251, pp.144-152.

Khatri, K., Goyal, A.K., Gupta, P.N., Mishra, N., Mehta, A. and Vyas, S.P. (2008) 'Surface modified liposomes for nasal delivery of DNA vaccine', Vaccine, Vol. 26, pp.2225-2233.

Kurita, K., Kaji, Y., Mori, T. and Nishiyama, Y. (2000) 'Enzymatic degradation of $\beta$-chitin: susceptibility and the influence of deacetylation', Carbohydrate Polymers, Vol. 42, pp.19-21.

Lowry, O., Rosebrough, N., Farr, A. and Randall, R. (1951) 'Protein measurement with the Folin phenol reagent', Journal of Biological Chemistry, Vol. 193, pp.265-275.

McNeela, E.A., Jabbal-Gill, I., Illum, L., Pizza, M., Rappuoli, R. and Podda, A. (2004) 'Intranasal immunization with genetically detoxified diphtheria toxin induces $\mathrm{T}$ cell responses in humans: enhancement of Th2 responses and toxin-neutralizing antibodies by formulation with chitosan', Vaccine, Vol. 22, pp.909-914.

Mills, K.H., Cosgrove, C., McNeela, E.A., Sexton, A., Giemza, R. and Jabbal-Gill, I. (2003) 'Protective levels of diphtheria-neutralizing antibody induced in healthy volunteers by unilateral priming-boosting intranasal immunization associated with restricted ipsilateral mucosal secretory immunoglobulin A', Infection and Immunity, Vol. 71, pp.726-732.

Mishra, D., Mishra, P.K., Dubey, V., Dabadghao, S. and Jain, N.K. (2007) 'Evaluation of uptake and generation of immune response by murine dendritic cells pulsed with hepatitis B surface antigen-loaded elastic liposomes', Vaccine, Vol. 25, pp.6939-6944.

Muller, R.H., Jacobs, C. and Kayser, O. (2001) 'Nanosuspensions as particulate drug formulations in therapy: Rationale for development and what we can expect for the future', Advanced Drug Delivery Reviews, Vol. 47, pp.3-19.

Nishimura, K., Ishihara, C., Ukei, S., Tokura, S. and Azuma, I. (1986) 'Stimulation of cytokine production in mice using deacetylated chitin', Vaccine, Vol. 4, pp.151-156.

Nishimura, K., Nishimura, S., Nishi, N., Saiki, I., Tokura, S. and Azuma, I. (1984) 'Immunological activity of chitin and its derivatives', Vaccine, Vol. 2, pp.93-99.

Onishi, H. and Machida, Y. (1999) 'Biodegradation and distribution of water-soluble chitosan in mice', Biomaterials, Vol. 20, pp.175-182.

Peek, L.J., Middaugh, C.R. and Berkland, C. (2008) 'Nanotechnology in vaccine delivery', Advanced Drug Delivery Reviews, Vol. 60, pp.915-928.

Ravi Kumar, M.N.V., Muzzarelli, R.A.A., Muzzarelli, C., Sashiwa, H. and Domb, A.J. (2004) 'Chitosan chemistry and pharmaceutical perspectives', Chemical Reviews, Vol. 104, pp.6017-6084.

Read, R.C., Naylor, S.C., Potter, C.W., Bond, J., Jabbal-Gill, I. and Fisher, A. (2005) 'Effective nasal influenza vaccine delivery using chitosan', Vaccine, Vol. 23, pp.4367-4374.

Saupe, A., McBurney, W., Rades, T. and Hook, S. (2006) 'Immunostimulatory colloidal delivery systems for cancer vaccines', Expert Opinion on Drug Delivery, Vol. 3, pp.345-354.

Seferian, P.G. and Martinez, M.L. (2001) 'Immune stimulating activity of two new chitosan containing adjuvant formulations', Vaccine, Vol. 19, pp.661-668.

Singla, A.K. and Chawla, M. (2001) 'Chitosan: some pharmaceutical and biological aspects - an update', Journal of Pharmacy and Pharmacology, Vol. 53, pp.1047-1067.

Van der Lubben, I.M., Verhoef, J.C., Borchard, G. and Junginger, H.E. (2001) 'Chitosan for mucosal vaccination', Advanced Drug Delivery Reviews, Vol. 52, pp.139-144. 
Varum, K.M., Holme, H.K., Izume, M., Stokke, B.T. and Smidsrod, O. (1996) 'Determination of enzymatic hydrolysis specificity of partially N-acetylated chitosans', Biochemica et Biophysica Acta, Vol. 1291, pp.5-15.

Zaharoff, D.A., Rogers, C.J., Hance, K.W., Schlom, J. and Greiner, J.W. (2007) 'Chitosan solution enhances both humoral and cell-mediated immune responses to subcutaneous vaccination', Vaccine, Vol. 25, pp.2085-2094. 